\title{
Therapeutic Dermoscopy to Facilitate Detection and Extraction of Foreign Bodies
}

\author{
Sody A. Naimer, MD
}

Foreign bodies are occasionally seen by family physicians. Plantar foreign bodies in particular pose a special challenge because they involve weight-bearing regions that are difficult to access. If left undetected long enough, these may lead to hospitalization, surgery, or even longstanding complications such as tumors, contractures, infections, and chronic ulcers. Dermoscopy of the cutaneous surface allows early detection of indwelling foreign bodies with a far greater degree of accuracy than with the naked eye. Furthermore, use of a polarized dermatoscope provides ideal illumination and 3-dimensional visualization of the involved site, and facilitates extraction of the penetrating object. This technique could be used for similar injuries involving other body surfaces. (J Am Board Fam Med 2017;30:374-376.)

Keywords: Dermoscopy, Foreign Bodies, Family Physicians

Foreign bodies are occasionally seen by family physicians. Plantar foreign bodies in particular pose a great challenge because they involve weight-bearing surfaces in an awkward location. ${ }^{1}$ Despite the seemingly benign character of an injury caused by stepping on a thorn or an unrecognized sliver of glass finding its way deep into the thick epidermal layer of the plantar skin, such events may lead to hospitalization, surgery, or even long-term complications such as tumors, contractures, infections, and chronic ulcers. ${ }^{2-6}$ If ignored, imaging may be needed to locate the penetrating object. ${ }^{7,8}$ It is assumed that clinicians would welcome a technique enabling prompt diagnosis and removal of most foreign bodies before the onset of difficult-to-treat complications.

The dermatoscope has attained growing popularity among family physicians. Its status has been

This article was externally peer reviewed.

Submitted 22 April 2016; revised 23 December 2016; accepted 23 January 2017.

From the Department of Family Medicine, Siaal Research Center for Family Medicine and Primary Care, Division of Community Health, Faculty of Health Sciences, Ben-Gurion University of the Negev, Beer-Sheva, Israel; and the Eilon Moreh Clinic, Clalit Health Services, Shomron District, Lev Shomron, Israel.

Funding: none.

Conflict of interest: none declared.

Corresponding author: Sody A. Naimer, MD, Department of Family Medicine, Faculty of Health Sciences, Ben-Gurion University of the Negev, POB 653, Beer-Sheva 84105, Israel (E-mail: sodyna@clalit.org.il). quoted by specialists as acquiring "a role similar to the stethoscope of general practitioners, becoming an irreplaceable clinical tool for dermatologists." This instrument offers a marked contribution in performing challenging therapeutic procedures. It has already been reported to facilitate the identification of obscure conditions such as the detection and extraction of unidentified corneal foreign bodies or buried sutures. ${ }^{10,11}$

Dermoscopy is recommended whenever a lesion can be characterized better with ideal illumination coupled with magnified visualization. The benefit conferred by a polarized dermatoscope, beyond the original episcope, is excellent focal accommodation without actual adherence to the surface being examined, which opens the possibility to perform procedures during observation of the involved area. This sets the stage for "therapeutic dermoscopy." An adapter is available that attaches a standard smartphone to the eyepiece of the dermatoscope (3Gen DermLite), which allows the complete unit to be held with one hand and leaves the dominant hand free to function as the situation dictates. The smartphone camera contributes further magnification if the zoom option is activated. This tool digitally magnifies the image, permitting examination of the imaged object from a more comfortable position (Figure 1). Furthermore, without extra effort, the complete procedure can be documented as a video or a photograph. Therapeutic dermoscopy 
Figure 1. Examination with a polarized dermoscope mounted on a smartphone.

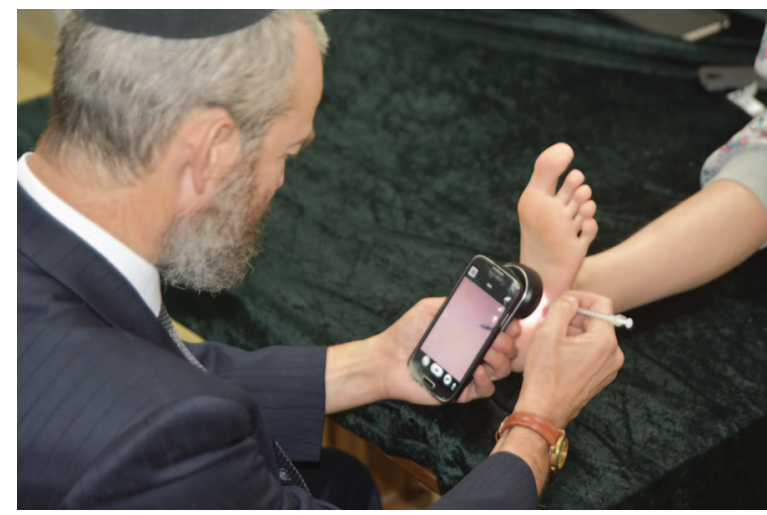

Figure 2. Unassisted appearance of the heel. A subtle break in the skin can be observed.

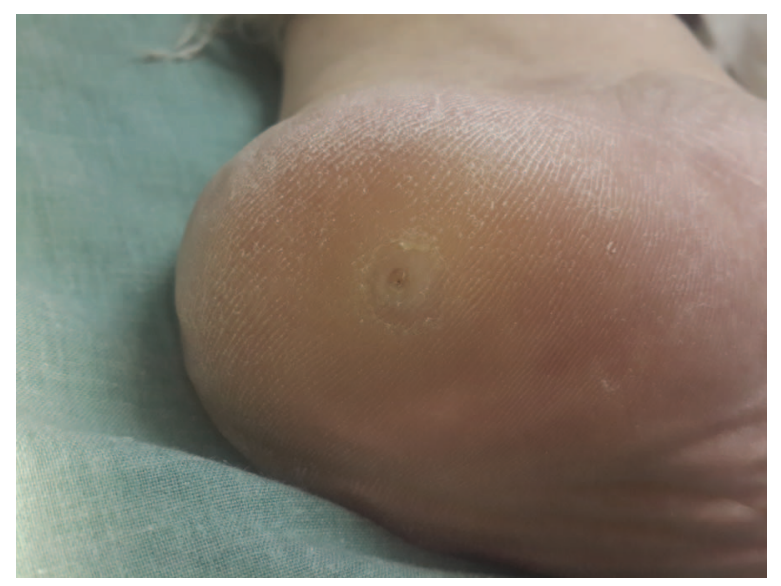

is a suitable technique for approaching a variety of small penetrating foreign bodies. Areas such as the heel and sole of the foot, where patient identification of a lesion is challenging, are particularly suitable for this technique. ${ }^{12}$ When dealing with plantar foreign bodies, immediate effective treatment becomes paramount to achieving complete clearance and optimizing patient satisfaction.

The following example demonstrates the contribution of therapeutic dermoscopy to the evaluation and treatment of a plantar foreign body.

\section{Illustrative Case}

A young man claimed an exquisitely painful heel lasting for the past month. He could not recall walking barefoot outside the household or ever stepping on anything sharp. Even close examination did not reveal the cause of his discomfort. Only a single dark dot on the plantar surface was appar-
Figure 3. Juxtamarginal pressure is applied adjacent to tip of the foreign body.

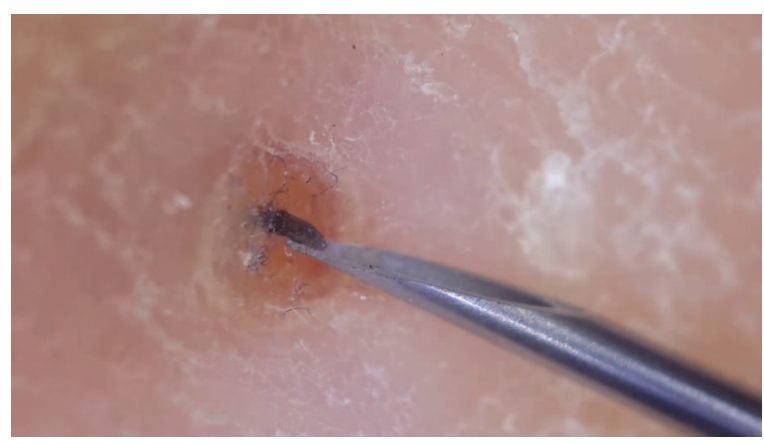

Figure 4. The full size of a rigid, spear-like body upon removal.

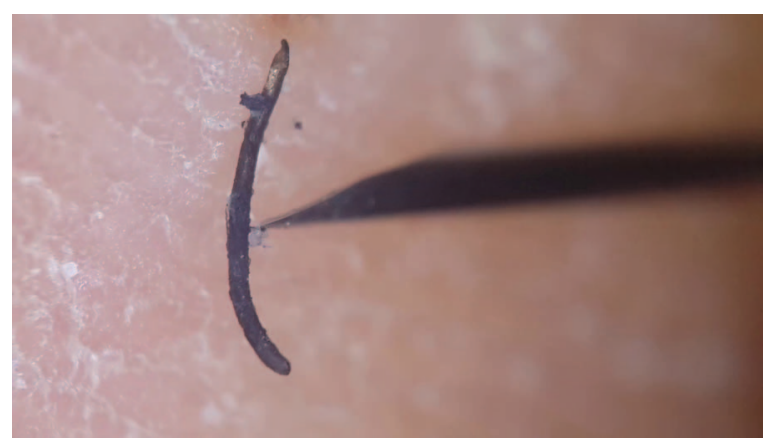

ent to the naked eye (Figure 2). Using the dermatoscope, visualization of the protruding tip of a pointed object resulted in the gradual extraction of a long foreign body (Figures 3 and 4). This procedure was completed without any incision. Fortunately, despite the long duration of the indwelling object, piercing perpendicularly deep into the soft tissue, no signs of infection were seen.

Dermoscopy seems to improve the ease and accuracy of locating a penetrating object, the removal of which eventually decreases patients' discomfort. This reduction of symptoms is particularly relevant in specific populations such as young children. The apparent simplicity of the procedure should save time, enabling it to be done during a routine visit.

The use of therapeutic dermoscopy is an acceptable and safe alternative for removing a foreign body. Therapeutic dermoscopy is advocated for use to evaluate and treat many diverse body surfaces, as it increases the likelihood of successful treatment of embedded epidermal objects while decreasing the risk of adverse sequelae from local tissue destruction. Other instances in which this technique has shown its worth for the family physician include 
delineating the surgical margins of neoplastic skin lesions, revealing ocular pathology, and identifying parasitic infestations. ${ }^{10,13}$

To see this article online, please go to: http://jabfm.org/content/ 30/3/374.full.

\section{References}

1. Eidelman M, Bialik V, Miller Y, Kassis I. Plantar puncture wounds in children: analysis of 80 hospitalized patients and late sequelae. Isr Med Assoc J 2003;5:268-71.

2. Dar TA, Sultan A, Hussain S, Dhar SA, Ali MF. Contracture of the third toe as a delayed presentation of a foreign body in the foot. Foot Ankle Spec 2011;4:298-300.

3. Di Chiacchio N, Wu SL, Gabriel FK, Bet DL, Di Chiacchio NG. Case for diagnosis. Puncture lesions with foreign body in the feet. An Bras Dermatol 2011;86:165-6.

4. Joseph WS, Le Frock JL. Infections complicating puncture wounds of the foot. J Foot Surg 1987;26 (1 Suppl):S30-3.

5. Barták V, Krátký Z, Popelka ml S. Tumour-like lesion of the forefoot due to a retained toothpick fragment. [Case report]. Acta Chir Orthop Traumatol Cech. 2014;81:152-4.
6. Woolfrey PG, Kirby RL. Hypodermic needles in the neuropathic foot of a patient with diabetes. CMAJ 1998;158:765-7.

7. Pattamapaspong N, Srisuwan T, Sivasomboon C, et al. Accuracy of radiography, computed tomography and magnetic resonance imaging in diagnosing foreign bodies in the foot. Radiol Med 2013; 118:303-10.

8. Nwawka OK, Kabutey NK, Locke CM, Castro-Aragon I, Kim D. Ultrasound-guided needle localization to aid foreign body removal in pediatric patients. J Foot Ankle Surg 2014;53:67-70.

9. Lallas A, Giacomel J, Argenziano G, et al. Dermoscopy in General Dermatology: Practical Tips for the Clinician. Br J Dermatol 2014;170:514-26.

10. Naimer SA, Urkin Y. Enhancement of pediatric ophthalmologic diagnosis with hand held polarized dermatoscopy. Clin Pediatr 2014;53:579-84.

11. Naimer SA. Prevention and Identification of Retained Sutures. J Am Acad Dermatol 2014;70:e57-8.

12. Naimer SA, Urkin Y. Expanded Use of the Otoscope and Dermatoscope in primary Care. Int J Med \& Med Sci 2013;5:535-45.

13. Naimer SA, Mumcuoglu KY. Diagnosis of ophthalmomyiasis externa by dermatoscopy. Dermatol Pract \& Conc 2014, 4(4): 85-7. 